BIOMEDICA

Vol. 3 No. 1 y $2-1983$

ARTICULOS ORIGINALES

\title{
URETRITIS MASCULINA ESTUDIO PROSPECTIVO DE 254 CASOS
}

\author{
FEDERICO DIAZ G. *
}

\begin{abstract}
La uretritis no gonocócica (UNG) fue la forma predominante $(78,0 \%$ ) en una serie de 254 hombres con síndrome de uretritis, estudiados prospectivamente en 1982. La Trichomonas vaginalis estuvo presente en el $4,0 \%$ de los casos de UNG.
\end{abstract}

Con base en la comparación entre los pacientes de uretritis gonocócica (UG) y los de UNG, puede proponerse un perfil de los segundo que oriente al médico en su sospecha diagnóstica: mayor probabilidad de no hallar secreción uretral o de que ésta sea escasa y de las modalidades acuosa, mucoide o mixta; también mayor probabilidad de un período de incubación por encima de 9 días y de una evolución superior a un mes.

A pesar de tales indicios hay mucha superposición clínica entre las dos formas de uretritis por lo que sigue siendo valedera la recomendación de recurrir al Gram como procedimiento confiable y económico para establecer el diagnóstico diferencial.

Se llama la atención hacia la necesidad de disponer de datos sobre uretritis en otros grupos socioeconómicos y de investigar aspectos como la frecuencia en nuestro medio del síndrome de uretritis postgonocócica (UPG).

\section{INTRODUCCION}

En un estudio previo (1) se analizaron retrospectivamente 1.557 pacientes con diagnóstico comprobado de uretritis; se halló un franco predominio de la forma no gonocócica (UNG) sobre la gonocócica (UG); la secreción uretral era escasa en la primera y abundante en la segunda; las modalidades de secreción acuosa, mucoide y mixta solo estuvieron presentes en pacientes de UNG; el hallazgo de Trichomonas vaginalis en el sedimento de la primera porción de orina matinal fue excepcional $(0,8 \%)$. Fue evidente la necesidad de hacer estudios prospectivos para refinar la información y conocer otros aspectos de la enfermedad en nuestro medio. Este informe resume la experiencia del año 1982, en 254 pacientes.

\section{MATERIALES Y METODOS}

El estudio se llevó a cabo en un laboratorio clínico que atiende personas remitidas por médicos que ejercen la medicina privada y prestan servicio a algunas instituciones oficiales.

* Profesor titular, Departamento de Microbiología y Parasitología, Facultad de Medicina, Universidad de Antioquia. 
Se definió como caso de uretritis el individuo remitido por disuria, secreción uretral o ambas y a quien se le demostraban más de 4 leucocitos PMN por campo de 1000 $\mathrm{X}$, como promedio de 5 campos de la secreción uretral, o 10 o más por campo de $400 \mathrm{X}$ al promediar 5 campos del sedimento de la primera porción de orina matinal (2).

Se obtuvieron datos demográficos (edad, estado civil, ocupación, nivel educativo), clínicos (período de incubación, demora para consultar después de la aparición del cuadro clínico y evolución de la enfermedad hasta la remisión para estudio microbiológico) y terapéuticos (antecedentes de antibioterapia en el mes inmediatamente anterior); se consignaron la modalidad e intensidad de la secreción uretral; la primera fue descrita por su aspecto macroscópico como purulenta, acuosa, mucoide o mixta (muco-purulenta, acuosa-purulenta. acuosa-mucoide); la segunda fue graduada según la siguiente escala: CERO cuando no había secreción; I: pequeña cantidad de secreción que solo sale por expresión del pene; II: cantidad moderada de secreción que solo sale por expresión del pene; III: secreción abundante que fluye espontáneamente.

Se buscó Trichomonas vaginalis en una gota de la secreción uretral disueita en solución salina; en ausencia de secreción, se utilizó para este propósito el sedimento de la primera porción de orina matinal.

La etiología de la uretritis (UG vs. UNG) se estableció sobre la base del resultado del Gram de la secreción o del sedimento urinario (primera porción de la orina matinal); se sabe $(3,4)$ que la presencia o ausencia de diplococos Gram negativos típicos intracelulares tiene alta sensibilidad y especificidad en la diferenciación de las dos formas de uretritis.

El significado de los hallazgos se analizó por medio de la prueba de X2.

\section{RESULTADOS}

Información demográfica:

Se incluyeron 187 pacientes de la práctica privada $(73,6 \%)$ y 67 de instituciones oficia- les $(26,3 \%)$; hubo 106 individuos casados (41,7\%); la edad de 213 pacientes $(83,3 \%$ ) estuvo comprendida entre los 20 y 39 años; catorce más $(5,5 \%)$ se encontraban entre 15 y 19 años; ninguno fue menor de 14 años; con respecto al nivel educativo 155 (61.\%) habían cursado algún nivel de educación postsecundaria y $80(31,5 \%)$ uno o más años de secundaria; cada una de las siguientes ocupaciones representó una quinta parte del grupo total: profesionales, empleados y estudiantes; el resto desempeñaba oficios muy diversos.

\section{Información clínica:}

El período de incubación fue de 9 días o menos en 123 individuos $(48,4 \%)$; de 10 o más en $84:(33,0 \%)$; los 47 restantes fueron incapaces de precisarlo.

La primera consulta médica la hicieron 182 pacientes $(71,7 \%)$ en la primera semana de la enfermedad; en la segunda semana otros $43(6,9 \%)$; los restantes acudieron al médico más tardiamente.

El tiempo transcurrido entra la primera consulta médica y el envío al laboratorio fue de un mes o menos en 133 individuos $(52,4 \%)$; de 2 a 24 meses en $69(27,2 \%)$ y superior a 24 meses en $15(5,9 \%) ; 37$ pacientes $(14,6 \%)$ no pudieron concretar esta información.

En 107 casos $(42,1 \%)$ había antecedentes de algún tipo de antibioterapia en el mes previo a la inclusión en este estudio.

\section{MODALIDADES DE LA SECRECION}

Hubo 60 pacientes $(23,6 \%)$ a quienes no se les detectó secreción uretral en el momento del examen; en 121 la secreción fue purulenta $(47,6 \%)$, en 15 acuosa $(5,9 \%)$, en 14 mucoide $(5,5 \%)$ y en 44 mixta $(17,3 \%)$; éstos se discriminaron así: 30 con secreción mucopurulenta; 8 en quienes fue acuosa-purulenta y 6 que la tuvieron acuosamucoide.

Intensidad de la secreción:

Sesenta pacientes $(23,6 \%)$ no tenían secreción (grado CERO); en 90 era de grado I 
$(35,4 \%)$; en 67 de grado II $(26,4 \%)$ y en 37 de grado III $(14,6 \%)$.

Etiología de la uretritis:

De acuerdo al Gram, 56 pacientes tuvieron UG $(22,0 \%)$ y 198 UNG $(78,0 \%)$.

\section{Infección con Trichomonas vaginalis}

Se halló el parásito en el 5,3\% de los casos estudiados con orina (3/57) y en el $2,5 \%$ de los analizados con secreción uretral (5/197); los 8 pacientes positivos representan el 3,1\% del grupo global y el 4,0\% de los 198 casos de UNG; en ningún caso hubo asociación de $T$. vaginalis y diplococos gram negativos.

Etiología e intensidad de la secreción:

La tabla No. 1 muestra que hubo abundante secreción (grado II y III) en el $73,2 \%$ de los pacientes de UG mientras fue escasa (grado CERO y I) en el $68,2 \%$ de los diagnosticados como UNG. La diferencia fue significativa $(\mathrm{p}<0,001)$.

Etiología y modalidad de la secreción:

En la tabla No. 2 se ve que el $83,9 \%$ de los pacientes con UG y solo el $37,2 \%$ de los UNG tenían secreción purulenta; esa diferencia fue significativa $(\mathrm{p}<, 001)$; las secreciones acuosa, mucoide y mixta, colectivamente consideradas, se hallaron en $12.5 \%$ de las UG y en $33.4 \%$ de las UNG; esta diferencia también fue significativa $(p<0,001)$.

TABLA $N^{\circ}$ ।

\begin{tabular}{|c|c|c|c|c|c|}
\hline \multicolumn{8}{|c|}{ ETIOLOGIA E INTENSIDAD DE LA SECRECION } \\
EN 254 CASOS DE URETRITIS \\
\hline \multirow{8}{*}{ GRADO } & \multicolumn{3}{|c|}{ ETIOLOGIA } & TOTAL \\
& N2 & 8 & N2 & $\$$ & \\
\hline O - I & 15 & 26,8 & 135 & 68,2 & 150 \\
II - III & 41 & 73,2 & 63 & 31,8 & 104 \\
TOTAL & 56 & 100,0 & 198 & 100,0 & 254 \\
\hline
\end{tabular}

TABLA $N^{*} 2$

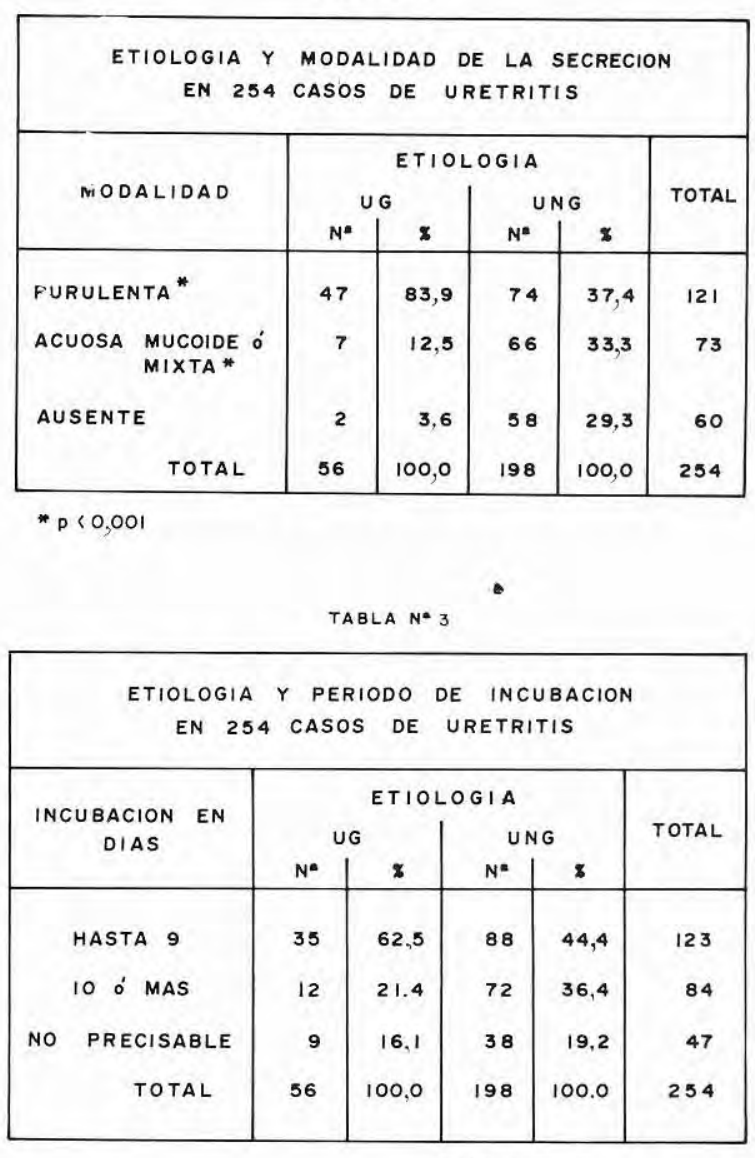

\section{Etiología y período de incubación:}

En la tabla No. 3 se aprecia que el $62.5 \%$ de las UG y solo el $44,4 \%$ de las UNG tuvieron un período de incubación de 9 días o menos; la diferencia fue significativa ( $p<0,02)$.

\section{Etiología y evaluación:}

La tabla No. 4 revela que los pacientes de UNG tenían más probabilidades que los de UG $(35,8 \%$ vs. $23,2 \%)$ de una enfermedad prolongada por más de un mes. La diferencia fue significativa $(\mathrm{p}<0,02)$.

No hubo diferencias en la precocidad de la primera consulta: $76,7 \%$ de los casos de UG y $69,9 \%$ de los de UNG acudieron al médico en la primera semana de la enfermedad. 
TABLA N*4

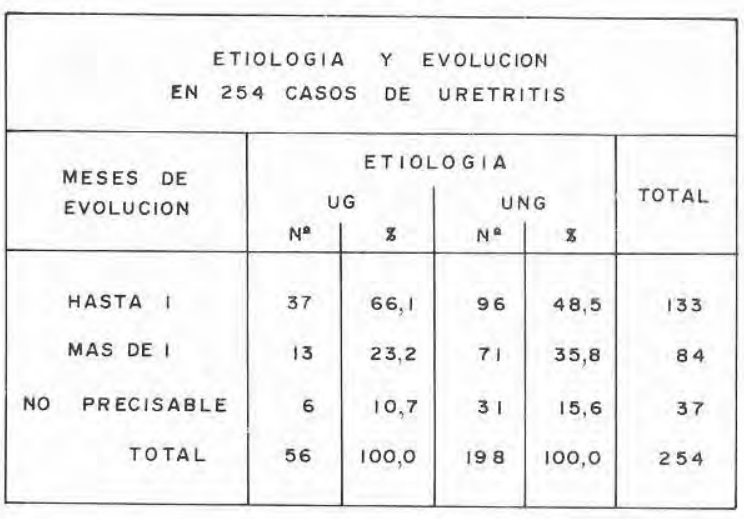

$p<0.02$

\section{DISCUSION}

La composición del grupo en cuanto a procedencia, ocupación y nivel educativo es simple reflejo del tipo de práctica médica en que se llevó a cabo este estudio; es concebible que los resultados del mismo no sean aplicables a otros grupos como el de pacientes de más bajos recursos económicos e inferior nivel educativo atendidos en los Centros Municipales de Salud; parecería de interés y relativamente sencillo adelantar estudios similares en tales instituciones.

En otros países (5) el principal incremento de las enfermedades sexualmente transmisibles (EST) ha ocurrido en el grupo de 15 a 19 años; el 5,5\% del presente grupo estaba en tal rango de edad; para saber si en Colombia se está dando la misma tendencia precisaría disponer de información sobre las varias EST en diversos estratos socioeconómicos.

La transmisión a la esposa o a la pareja sexual de los microorganismos causales de uretritis puede tener lugar durante el período de incubación; eso pudo haber ocurrido en los pacientes casados de esta serie $(41,7 \%)$; de ahí que deba insistirse en el tratamiento de la pareja; cabe la posibilidad de que una vez manifestada abiertamente la uretritis el paciente se abstenga transitoriamente de relaciones sexuales; este aspecto, que tiene importancia en la cadena de transmisión, debe ser motivo de investigación ulterior.

Dado el antecedente de antibioterapia en el mes previo al estudio microbiológico $\{42,1 \%$ de los pacientes $)$ puede especularse que algunas de las UNG eran de la variedad post-gonocócica (UPG); la frecuencia de ésta en Colombia es desconocida y sería importante estudiarla en pacientes con antecedente comprobado de UG tratados con antibióticos distintos de las tetraciclinas. Estudios previos han demostrado (6) que la UPG aparece en más del $90.0 \%$ de los hombres con gonorrea que son, además, positivos para Chlamydia trachomatis, cuando son tratados con gentamicina, ampicilina o espectinomicina: si se dan las mismas circunstancias pero con negatividad para la Chlamydia la frecuencia de UPG fluctúa entre el $17,0 \%$ y el $45,0 \%$.

En un estudio previo (1) se halló una frecuencia muy baja de trichomoniasis $(0,8 \%)$ en pacientes con uretritis; en la serie que se presenta el parásito estuvo presente en el $3,1 \%$ del grupo total $(4,0 \%$ de los pacientes de UNG); el hallazgo de la Trichomonas vaginal is fue más frecuente en los casos estudiados con la primera porción de la orina matinal $(5,3 \%)$ que cuando se practicó la búsqueda en la secreción uretral $(2,5 \%)$; sin embargo no puede hacerse con esta base la recomendación de preferir una u otra muestra; más bien la de realizar un estudio comparativo buscando el parásito en ambas muestras en todos los pacientes y, en lo posible, practicando cultivos para Trichomonas; sólo así podrá conocerse con certeza la importancia de la misma en síndromes uretrales.

Se confirmó el hallazgo de la serie previa (1) de que la UNG excede a la UG en este grupo socioeconómico; la proporción en esta serie fue de $3,5 / 1$; en Inglaterra (7) la frecuencia de UNG excedió a la de UG desde 1965 y en EE. UU. la proporción UNG/UG favoreció a la primera en 7 de los 13 trabajos revisados por Wiesner (7).

Con base en los datos de este estudio puede proponerse un perfil que permita al médico plantear el diagnóstico de UNG: la 
cantidad de secreción uretral es pequeña o no hay secreción; con mayor probabilidad que en el caso de la UG la secreción es acuosa, mucoide o mixta; el período de incubación tiende a ser más prolongado y mayor la probalidad de que las manifestaciones hayan estado presentes por más de un mes; sin embargo ninguno de estos criterios es absoluto y sigue siendo aconsejable recurrir al Gram de la secreción uretral o de la primera porción de la orina matinal para establecer más certeramente el diagnóstico.

Pueden proponerse para futuras investigaciones del tema de la uretritis, además de los mencionados, los siguientes aspectos: a) detalles de la antibioterapia previa; b) frecuencia de automedicación inicial o de prescripción por el farmacéuta; c) frecuencia con que el médico prescribe sin recurrir antes al estudio de laboratorio.

\section{SUMMARY}

Among 254 male patients with urethritis, prospectively studied in 1982 , a predominance was found $(78,0 \%)$ of the nongonococcal form (NGU); Trichomonas vaginalis was present in $4,0 \%$ of the NGU cases.

Based on the comparison between patients with gonoccoccal and nongonococcal urethritis a profile of the latter can be proposed to orient the physician's diagnostic suspicion: NGU patients have a greater probability of showing none or scarce secretion and of having the aqueous, mucoid or mixed (muco-purulent, aqueous-purulent, aqueous-mucoid) modalities of the secretion; also, they have a greater probability of an incubation period above 9 days and a duration of the disease above one month.

Despite these clues there is much overlapping between clinical features of the two forms of urethritis; therefore, the recommendation to routinely perform a Gram stain is still valid, as a reliable and economic precedure for establishing the differentation.

Information is required on urethritis in different colombian socioeconomic groups; also research is needed in areas such as the frequency of postgonococcal urethritis (PGU).

\section{BIBLIOGRAFIA}

1. Díaz, F. Uretritis masculina gonocócica y no gonocócica. Papel del Laboratorio Clínico en su diagnóstico microbiológico. Biomédica 2 (4), en prensa.

2. Desai, K., Robson, H.G. Comparison of the Gramstained urethral smear and first-voided urine sediment in the diagnosis of ningonococcal urethritis. Sex Transm Dis 9 (1): 21-25. 1982.

3. Goodhart, M.E., et al. Factors affecting the performance of smear and culture tests for the detection of Neisseria gonorrhoeae. Sex Trans. Dis 9 (2): 63-39, 1982.

4. Kraus, S.J. Semiquantitation of urethral palymorphonuclear leukocytes as objective evidence of nongonococcal urethritis. Sex Transm. Dis 9 (1) $52-55,1982$.

5. Lasagna, L. The VD epidemic. Philadelphia, Temple, 1975, p. 6.

6. Oriel, J.D. Chlamydia trachomatis and postgonococcal genital infection. IN: Hobson, D., Holmes, K.K. ed. Nongonococcal urethritis and related infections, Washington, American Society for Microbiology, 1977, pp. 230-232.

7. Wiesner, P.J.Selected aspects of the epidemiology of ningonococcal urethritis. IN: Hobson, D., Holmes, K.K. ed. Nongonococcal urethritis and related infections. Washington, American Society for Microbiology, 1977, pp. 9-14. 